\title{
ORIGINAL RESEARCH \\ Brain Iron Quantification in Mild Traumatic Brain Injury: A Magnetic Field Correlation Study
}

\begin{abstract}
E. Raz
J.H. Jensen

Y. Ge

J.S. Babb

L. Miles

J. Reaume

R.I. Grossman M. Inglese

BACKGROUND AND PURPOSE: Experimental studies have suggested a role for iron accumulation in the pathology of TBI. Magnetic field correlation MR imaging is sensitive to the presence of non-heme iron. The aims of this study are to 1) assess the presence, if any, and the extent of iron deposition in the deep gray matter and regional white matter of patients with MTBI by using MFC MR imaging; and 2) investigate the association of regional brain iron deposition with cognitive and behavioral performance of patients with $\mathrm{mTBI}$.

MATERIALS AND METHODS: We prospectively enrolled 28 patients with mTBI. Eighteen healthy subjects served as controls. The subjects were administered the Stroop color word test, the Verbal Fluency Task, and the Post-Concussion Symptoms Scale. The MR imaging protocol (on a 3T imager) consisted of conventional brain imaging and MFC sequences. After the calculation of parametric maps, MFC was measured by using a region of interest approach. MFC values across groups were compared by using analysis of covariance, and the relationship of MFC values and neuropsychological tests were evaluated by using Spearman correlations.

RESULTS: Compared with controls, patients with mTBI demonstrated significant higher MFC values in the globus pallidus $(P=.002)$ and in the thalamus $(P=.036)$. In patients with mTBI, Stroop test scores were associated with the MFC value in frontal white matter $(r=-0.38, P=.043)$.

CONCLUSIONS: MFC values were significantly elevated in the thalamus and globus pallidus of patients with $\mathrm{mTBI}$, suggesting increased accumulation of iron. This supports the hypothesis that deep gray matter is a site of injury in $\mathrm{mTBI}$ and suggests a possible role for iron accumulation in the pathophysiological events after mTBI.

ABBREVIATIONS: DAI = diffuse axonal injury; $E P I=$ echo-planar imaging; FSE = fast spin-echo; GCS = Glasgow Coma Scale; GE = gradient-echo; MFC = magnetic field correlation; MFI = microscopic field inhomogeneity; MPRAGE = magnetization-prepared rapid acquisition of gradient echo; $\mathrm{mTBI}=$ mild traumatic brain injury; PCS = postconcussive syndrome; PCSS = PostConcussion Symptoms Scale; ROI = region of interest; $\mathrm{TBI}=$ traumatic brain injury; VFT = Verbal Fluency Test
\end{abstract}

m TBI accounts for $90 \%$ of the new cases of head trauma diagnosed annually in the United States, ${ }^{1}$ with an annual incidence rate as high as $128 / 100,000^{2,3}$ and an estimated total lifetime annual cost of US\$17 billion. ${ }^{4} \mathrm{Up}$ to $15 \%$ of patients with mTBI will suffer a PCS $^{3}$ characterized by persistent disabling physical (headache, dizziness), cognitive (memory loss, attention deficit), and emotional/behavioral (depression, anxiety) symptoms. Although the injury associated with moderate and severe trauma is usually evident on conventional MR imaging as hemorrhagic lesions or contusions, in most cases of mTBI both CT and MR imaging scans do not show obvious lesions. ${ }^{5}$ Likewise, although DAI is a common finding in moderate and severe TBI, in case of $\mathrm{mTBI}$, not only there is a

Received January 10, 2011; accepted after revision February 26.

From the Department of Radiology (E.R., J.H.J., Y.G., J.S.B., L.M., J.R., R.I.G.), New York University School of Medicine, New York, New York; Department of Neurological Sciences (E.R.), Sapienza University of Rome, Rome, Italy; and Department of Neurology and Radiology (M.I.), Mount Sinai School of Medicine, New York, New York.

This study was supported by the National Institute of Health grants R01 NS039135 and R01 NS051323.

Please address correspondence to Matilde Inglese, MD, PhD, Department of Neurology, Mount Sinai School of Medicine, Annenberg 14-89, Box 1137, One Gustave L. Levy Place, New York, NY 10029; e-mail: matilde.inglese@mssm.edu

Indicates open access to non-subscribers at www.ajnr.org

http://dx.doi.org/10.3174/ajnr.A2637 paucity of classic DAI-related MR findings but also the few injuries detected are focal and cannot justify the stereotypical global PCS symptoms.

This has been attributed to the inability of routine imaging to reveal the presence of microscopic brain damage that has been reported by studies in experimental models and autoptic tissue of patients with mTBI. ${ }^{6}$ Animal studies also have shown that mild head injury can cause oxidative stress injury and subtle axonal damage in the absence of gross focal lesions. ${ }^{7,8}$ In addition, trauma-induced increased blood-brain barrier permeability ${ }^{9}$ and hemoglobin degradation products can be detected in the absence of focal lesions, ${ }^{10}$ suggesting a mechanism for excessive iron deposition in TBI. A role for iron accumulation in the pathology of TBI has been suggested by a recent correlative MR imaging-histologic study ${ }^{11}$ that examined the brains of mice after a controlled cortical impact injury. Interestingly, a T2 hypointensity, which showed increased staining of iron at the histologic examination, was observed in the thalamus ipsilateral to the acute cortical impact injury. In the postacute period, astrogliosis and microgliosis were observed in the ipsilateral thalamus, raising the possibility that iron accumulation may trigger the pathologic events after TBI. ${ }^{11}$

MFC is a recently introduced MR imaging metric ${ }^{12-14}$ that has a direct relationship to the MFIs created by spatial variations in magnetic susceptibility, such as those generated by 
macroscopic structures (air, cavities, large veins, or bone) and iron-rich cells, with length scales ranging roughly from 1 to $100 \mu \mathrm{m}$. MFC MR imaging has been applied to the study of a patient with aceruloplasminemia, ${ }^{12}$ showing increased values in the globus pallidus, thalamus, and frontal white matter, consistent with the increased brain iron concentration, typical of this neurologic disease. An MFC MR imaging study of patients with multiple sclerosis also has demonstrated increased MFC index in the deep gray matter, ${ }^{15}$ plausibly reflecting iron deposition secondary to the presence and number of white matter lesions typical of the disease. The aims of our study were to 1) assess the presence, if any, and the extent of iron deposition in the gray matter and regional white matter of patients with mTBI by using MFC as an iron biomarker; and 2) investigate the association of regional brain iron deposition with cognitive and behavioral performance of patients with mTBI.

\section{Materials and Methods}

\section{Patients}

The study population was a series of patients with a well-documented clinical history of mTBI recruited prospectively from our hospital records and emergency department. All patients experienced a closed head injury resulting in an alteration of mental status. Inclusion criteria were 1) diagnosis of mTBI and 2) age between 18 and 60 years. mTBI was defined according to established criteria ${ }^{16}:$ 1) posttraumatic amnesia not $>24$ hours, 2) GCS score between 13 and 15, and 3) loss of consciousness of approximately $\leq 30$ minutes. Exclusion criteria were 1) a history of other neurologic disease, 2) psychiatric disturbance, 3) a history of substance or alcohol abuse, and 4) contraindications to performing MR imaging. Accordingly, 2 patients were excluded due to history of alcohol abuse, and 2 patients had contraindications to performing MR imaging. The remaining $28 \mathrm{pa}-$ tients were included in the study. A board-certified neurologist clinically evaluated all patients, and a clinical psychologist performed the neuropsychologic tests. The 28 patients had a mean age of $35.6 \pm 10.4$ years; there were 9 women and 19 men. The mean time interval between the trauma and the MR imaging examination was $559 \pm 803$ days. The GCS score was 13 in 4 patients, 14 in 9 patients, and 15 in 15 patients. Eighteen healthy volunteers ( 7 women and 11 men; mean age, $34.6 \pm 7.4$ years) were enrolled as controls. The study was approved by the institutional review board of our university, and it was in compliance with the Health Insurance Portability and Accountability Act. Written informed consent was obtained from all the participants.

\section{Neuropsychological Tests}

All subject were administered the Stroop test ${ }^{17}$ and the Delis-Kaplan Executive Function System VFT, ${ }^{18}$ but only the patients were administered the PCSS ${ }^{19}$; all tests were administered within 24 hours of undergoing the MR imaging scan. The Stroop test is a test of executive function that assesses the ability to inhibit habituated responses. The VFT is also a test of executive function, assessing verbal fluency, speed of information processing, and mental flexibility. For ease of comparison, scores for the Stroop and VFT were transformed into $z$ scores. Patients who were at or below the fifth percentile $(-1.6$ SDs below the normative mean) of performance on each test compared with controls were categorized as cognitively impaired. The PCSS is a selfreport questionnaire that assesses the severity of 19 symptoms asso- ciated with PCS/mild cognitive impairment. Items are rated on a Likert scale, indicating severity of the symptom, from 0 (none) to 6 (severe).

\section{MR Imaging Acquisition}

All subjects underwent MR imaging on a 3T scanner (Tim Trio; Siemens Medical Solutions, Erlangen, Germany). The body coil was used for signal intensity transmission, and the manufacturer-provided 12channel head coil designed for parallel imaging was used for signal intensity reception. Section orientation parallel to the subcallosal line was assured by acquiring a multiplanar T1-weighted localizer at the beginning of each study. The MR imaging protocol included the following sequences:

1. Axial T2-weighted FSE sequence on 22 sections $(\mathrm{TR}=5700 \mathrm{~ms}$, $\mathrm{TE}=113 \mathrm{~ms}$, matrix $=487 \times 635, \mathrm{FOV}=320 \times 320 \mathrm{~mm}^{2}$, section thickness $=3 \mathrm{~mm}$, intersection gap $=3 \mathrm{~mm}$, acceleration factor $=2$, scanning time $=3$ minutes 15 seconds.

2. Sagittal 3D T1 MPRAGE sequence on 160 contiguous sections $\left(\mathrm{TR}=2300 \mathrm{~ms}, \mathrm{TE}=3 \mathrm{~ms}, \mathrm{TI}=900 \mathrm{~ms}\right.$, flip angle $=9^{\circ}$, matrix $=$ $487 \times 635$, FOV $=240 \times 240 \mathrm{~mm}^{2}$, section thickness $=1 \mathrm{~mm}$, intersection gap $=0 \mathrm{~mm}$, acceleration factor $=2$, scanning time $=4$ minutes 21 seconds).

3. Axial $\mathrm{T} 2 \mathrm{GE}$ sequence on 22 sections $(\mathrm{TR}=800 \mathrm{~ms}$, TE $=20 \mathrm{~ms}$, flip angle $=15^{\circ}$, matrix $=487 \times 635, \mathrm{FOV}=320 \times 320 \mathrm{~mm}^{2}$, section thickness $=3 \mathrm{~mm}$, intersection gap $=2 \mathrm{~mm}$, acceleration factor $=2$, scanning time $=3$ minutes 35 seconds).

4. Axial MFC by using a segmented (4 shot) EPI asymmetric echo sequence on 48 sections $(\mathrm{TR}=5130 \mathrm{~ms}, \mathrm{TE}=40 \mathrm{~ms}$, matrix $=128 \times$ 128 , flip angle $=90^{\circ}, \mathrm{FOV}=256 \times 256 \mathrm{~mm}^{2}$, bandwidth $=1346$ $\mathrm{Hz} /$ pixel, section thickness $=1.7 \mathrm{~mm}$, intersection gap $=0 \mathrm{~mm}[\mathrm{re}-$ sulting in isotropic voxels], scanning time $=6$ minutes 8 seconds). Images were acquired with refocusing pulse time shifts of $t_{s}=0,-4$, and $-16 \mathrm{~ms}$, where the negative sign indicates a reduction of the interval between the initial $90^{\circ}$ excitation pulse and $180^{\circ}$ refocusing pulse from the usual spin-echo value of TE/2. For each set of imaging parameters, 4 images were acquired to increase the signal intensityto-noise ratio. ${ }^{12}$

\section{Postprocessing}

The MR images were transferred off-line and processed by an MR expert and a neuroradiologist; T2-FSE, T1-MPRAGE, and T2-GE were reviewed for any area of altered signal intensity. The postprocessing of MFC was performed in Matlab 7.0 (MathWorks, Natick, Massachusetts), by using the SPM 5 software (Wellcome Department of Cognitive Neurology, London, England) as described in detail previously. ${ }^{12,13}$ For a biologic tissue in an applied magnetic field, the MFC may be defined by the following equation:

$$
\operatorname{MFC}\left(\left|t-t^{\prime}\right|\right)=\gamma^{2}\left\langle\delta B(t) \delta B\left(t^{\prime}\right)\right\rangle
$$

with $\delta B(t)$ being the magnitude difference between the total and background fields for a water molecule at a time $t$ and with $\gamma$ being the proton gyromagnetic ratio. ${ }^{12,13}$ In the equation, the field product within brackets is averaged over all water molecules within a voxel. Because of time translation invariance, the MFC depends only on the time difference. Thus, the MFC provides a quantitative means of characterizing MFIs. After coregistration and smoothing, the 4 repetitions were averaged for each refocusing shift and the parametric maps of the MFC were generated, determined from a least-squares nonlinear equation by using in-house Matlab scripts. ${ }^{12,13}$ Using Im- 


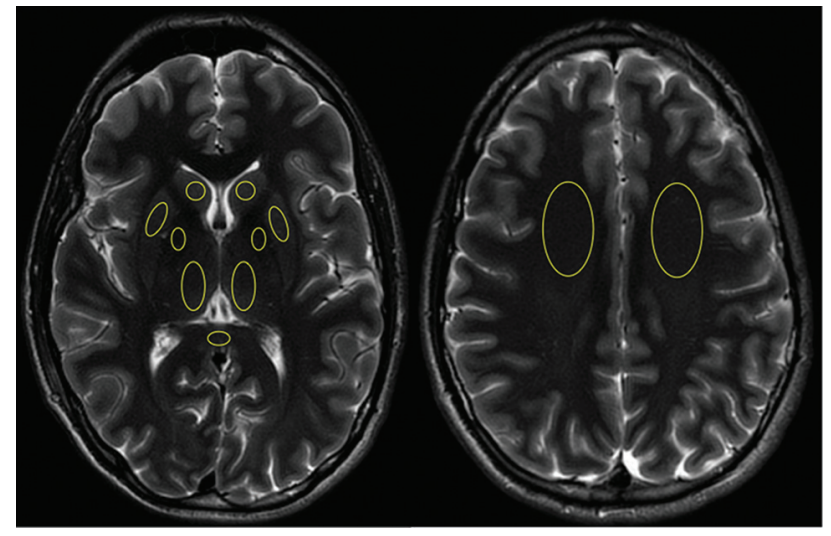

Fig 1. Representative of the ROls drawn on 2 sample $T 2$ images at the level of basal ganglia and centrum semiovale.

\begin{tabular}{lc}
\hline Table 1: Clinical symptoms in the patient group & \\
\hline & $\begin{array}{c}\text { No. of } \\
\text { Pymptom }\end{array}$ \\
\hline Blurry vision & 1 \\
Nausea & 9 \\
Memory loss & 8 \\
Dizziness & 7 \\
Photophobia & 6 \\
Neck stiffness & 5 \\
Loss of smell & 4 \\
Sleep disturbance & 3 \\
Headaches & 2 \\
Pain & 1 \\
Numbness & 1 \\
Tingling & 1 \\
\hline
\end{tabular}

ageJ (http://rsbweb.nih.gov/ij/index.html), standard ellipse-shaped ROIs of the following brain regions were then outlined on T2 images and applied on 3 consecutive coregistered MFC maps: the caudate nucleus, thalamus, putamen, globus pallidus, the frontal white matter bilaterally and the splenium of the corpus callosum. The standard ellipse-shaped ROIs (Fig 1) were placed by a neuroradiologist (E.R.) who adjusted the size and orientation depending on the brain area analyzed (eg, globus pallidus, thalamus, caudate nucleus) on 3 contiguous sections bilaterally. The mean MFC value (unit of measure = $\mathrm{s}^{-2}$ ) and $\mathrm{SD}$ of the ROIs were calculated and then averaged over sections and brain side.

\section{Statistical Analysis}

Mixed model analysis of covariance was used to compare subject groups in terms of regional MFC values adjusted for age and sex. Specifically, the regional MFC measures were modeled as functions of subject group, age, and sex. The correlation structure imparted by the inclusion of multiple MFC values per subject was modeled by assuming data to be correlated only when acquired from the same subject and by allowing the error variance to differ across comparison groups (to eliminate the assumption of variance homogeneity). Spearman rank correlations were used to assess the relationship of MFC values with the Stroop and VFT $z$ scores, the PCSS scores, and the elapsed time from injury. Because Stroop, VFT, PCSS, and elapsed time from injury are subject-level factors, each was correlated with MFC values represented for each subject as an average. All reported $P$ values are 2-sided, and statistical significance is defined as $P<.05$. SAS 9.0 (SAS Institute, Cary, North Carolina) was used for all computations.
Table 2: Average MFC values $\left(\mathrm{s}^{-2}\right) \pm \mathrm{SDs}$ in healthy controls and patients with mild traumatic brain injury

\begin{tabular}{lllc}
\hline Brain Region & Controls & Patients & $P$ \\
\hline Caudate & $269 \pm 146$ & $326 \pm 147$ & .111 \\
Thalamus & $149 \pm 59$ & $181 \pm 65$ & $.036^{*}$ \\
Globus pallidus & $689 \pm 252$ & $873 \pm 327$ & $.002^{*}$ \\
Putamen & $430 \pm 166$ & $493 \pm 234$ & .331 \\
Splenium & $243 \pm 190$ & $210 \pm 140$ & .638 \\
Frontal lobe white matter & $273 \pm 176$ & $203 \pm 153$ & .130 \\
\hline
\end{tabular}

Note:-Asterisk indicates statistically significant comparisons.

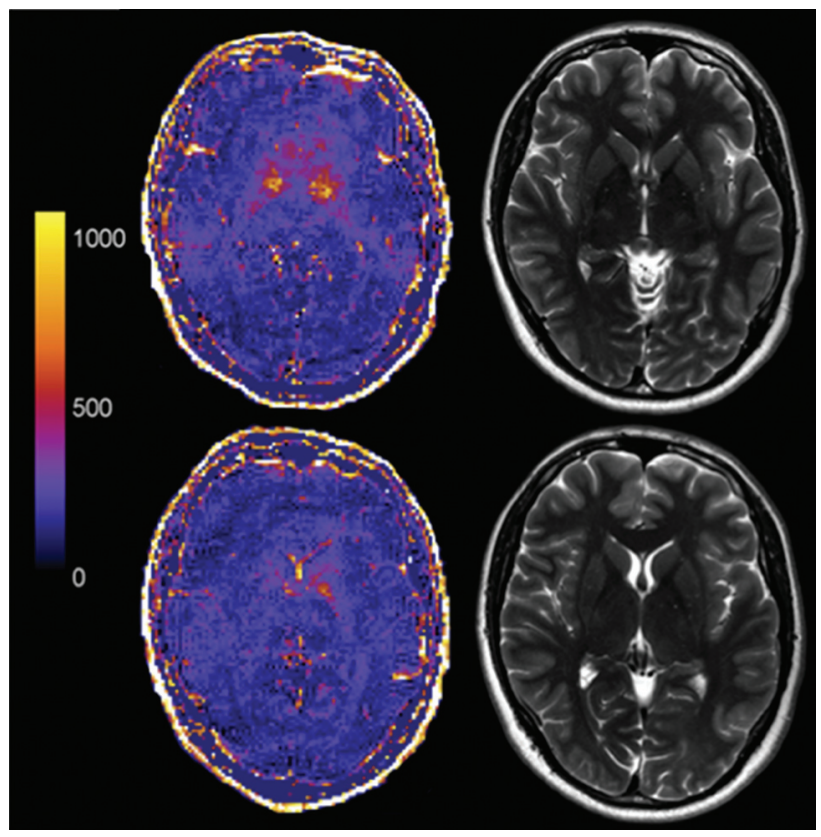

Fig 2. Representative MFC color maps and correspondent $\mathrm{T} 2$ images of 2 brain sections at the level of globus pallidus and thalamus in a 42-year-old male patient with mTBI. The patient complained of neck stiffness; his GCS score was 15, and the conventional MR imaging scan was normal. Note the clearly increased MFC in the deep gray matter and the correspondent normal T2 images. MFC values are reported in $\mathrm{s}^{-2}$.

\section{Results}

All patients with mTBI suffered blunt head trauma (eg, motor vehicle crash, bicycle accident, fall, assault). Most of the patients experienced residual symptoms as detailed in Table 1. T2-FSE and T1-MPRAGE images showed no area of altered signal intensity either in patients or in healthy volunteers. T2-GE showed a dotlike hypointensity in the fusiform gyrus of 1 patient. Mean and SDs of MFC values in patients with mTBI and controls are reported in Table 2. Compared with healthy volunteers, patients demonstrated significantly higher MFC values in the globus pallidus $(P=.002)$ and the thalamus $(P=$ .036). Representative MFC maps with corresponding T2 images from 2 brain sections of 2 patients with mTBI (Figs 2 and 3) and a healthy subject (Fig 4) are shown.

The mean PCSS score of patients with mTBI was $34 \pm 25$. The mean Stroop color word test and the VFT test $z$ scores were significantly lower $(P=.018$ and $P=.012$, respectively) in patients with mTBI $(-0.82 \pm 1.82$ and $-0.28 \pm 1.35$, respectively) than in healthy controls $(0.43 \pm 0.42$ and $0.93 \pm$ 1.23 , respectively). Seven of 28 patients $(25 \%)$ were deemed impaired on the Stroop test, and 7 of 28 patients (25\%) on the 


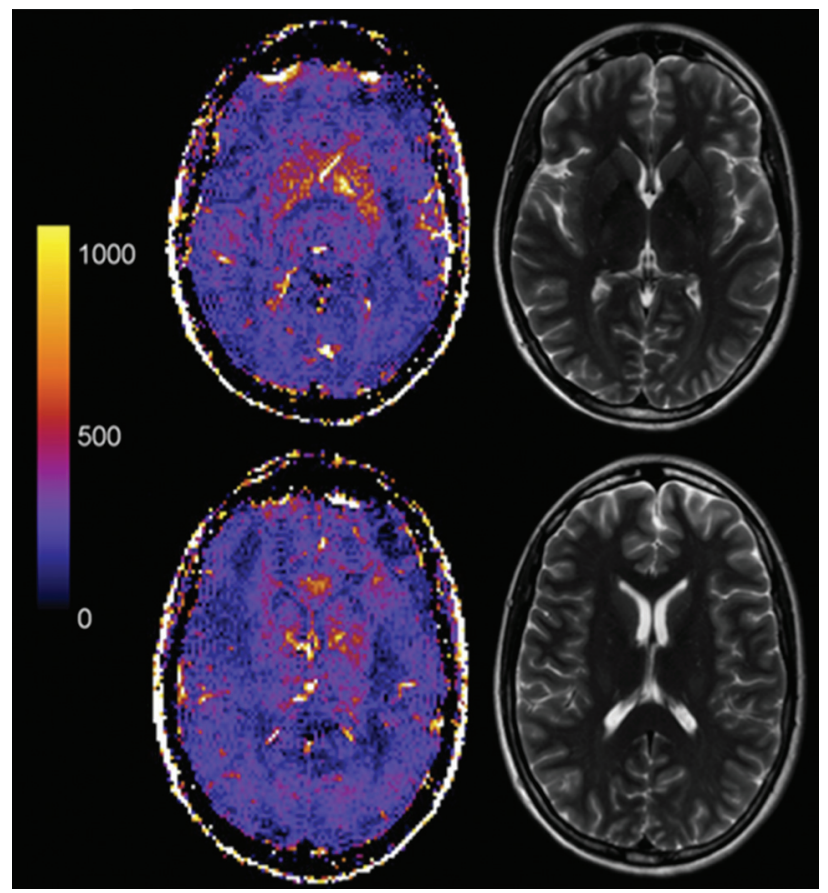

Fig 3. Representative MFC color maps and correspondent $\mathrm{T} 2$ images of 2 brain sections at the level of globus pallidus and thalamus in a 25 -year-old male patient with mTBI. The patient complained of headache, neck stiffness, photophobia, and nausea; his GCS score was 13 , and the conventional MR imaging scan was normal. Note the clearly increased MFC in the deep gray matter and the correspondent normal T2 images. MFC values are reported in $\mathrm{s}^{-2}$

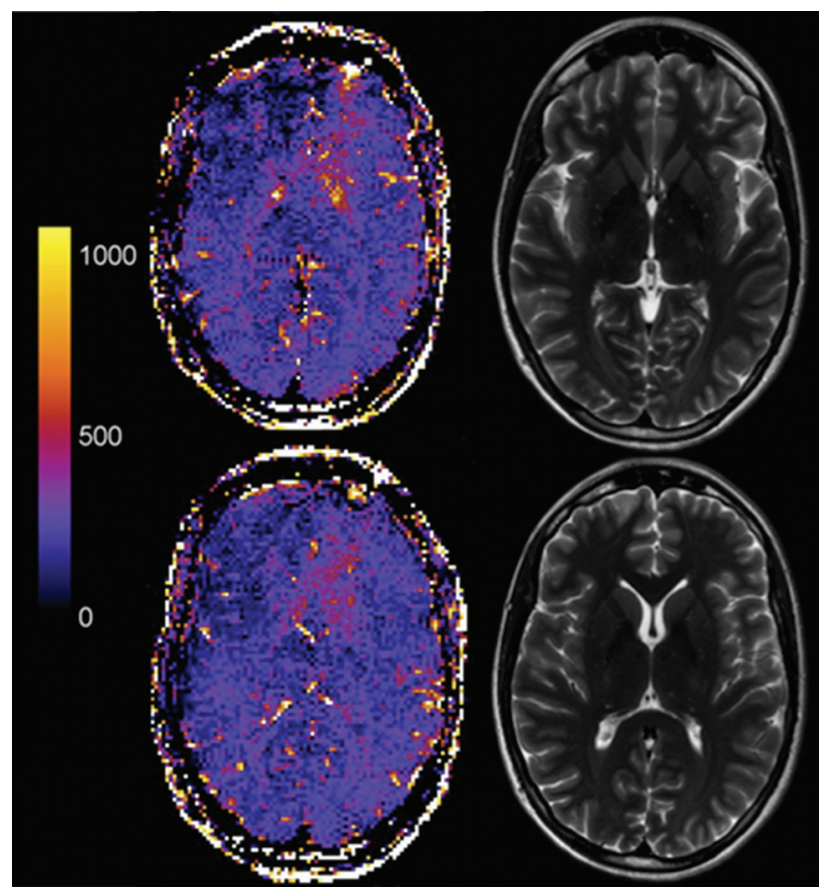

Fig 4. Representative MFC color maps and correspondent $\mathrm{T} 2$ images of 2 brain sections at the level of globus pallidus and thalamus in a 32-year-old male healthy volunteer. MFC values are reported in $\mathrm{s}^{-2}$.

VFT test score. In patients with mTBI, the Stroop test scores were inversely associated with the MFC value in frontal white matter $(r=-0.38, P=.043)$. PCSS and VFT tests did not show significant association with the MFC value of any of the examined brain regions $(P>.07)$. In controls, there were no significant correlations between MFC values and Stroop or VFT test scores $(P>.16)$.

Finally, no correlations were found between the MFC value of any of the examined regions and the interval from trauma $(P>.13)$.

\section{Discussion}

Using a newly developed MFC method, we have observed increased MFC values in the deep gray matter of patients with mTBI, suggesting a role for iron in the pathophysiologic cascade of events after the traumatic insult. In addition, our findings support the hypothesis that not only white matter regions and the gray-white matter junction are injured by the shear stress forces associated to the traumatic event but also subcortical gray matter regions such as the thalamus and the globus pallidus.

We used for our study the MFC sequence: although the conventional method for exploiting the effect of MFIs has been to measure the relaxation rates $\mathrm{R} 2, \mathrm{R} 2{ }^{*}$, and $\mathrm{R} 2^{\prime}$, this has possible disadvantages; in particular $\mathrm{R} 2$ and $\mathrm{R} 2^{\star}$ are influenced by other relaxation mechanisms such as dipolar interactions, whereas MFC depends only on MFIs and weakly on water diffusion. Although R2' can provide a better characterization of MFIs than R2 and R2*, the precise physical meaning of $\mathrm{R}^{\prime}$ is not well determined, and it is more dependent on the experimental technique. ${ }^{14}$ In contrast, MFC has a more direct relationship to the MFIs, allowing for a clearer physical interpretation. $^{12}$

Although less susceptible to direct damage, the deep gray matter nuclei are more sensitive to shear and strain damage because of the long fibers that originate or pass through them. ${ }^{3,20,21}$ The thalami and basal ganglia are the centrally located relay station for information throughout the brain, and they participate in communication among sensory, motor and associative areas; ${ }^{22}$ thus, damage to these structures can determine widespread functional impairments. ${ }^{23}$

The mechanism of the excessive accumulation of iron in deep gray matter in mTBI is not well known, and it is probably multifactorial. Iron is essential for many metabolic functions including the biosynthesis of neurotransmitters and formation of myelin. ${ }^{24}$ Abnormal iron deposition, however, can be injurious to the central nervous system ${ }^{25}$ by inducing oxidative stress that facilitates neuronal death and neurodegeneration. ${ }^{26}$ Whether accumulation of iron is the primary pathologic factor of the traumatic insult or a secondary effect due to diffuse axonal injury of white matter regions is uncertain; however, its toxic effects on lipids, carbohydrates, proteins, and nucleic acids are established. ${ }^{26}$ Animal studies have shown that mild head injury causes such oxidative stress injury and subtle axonal damage in the absence of gross focal lesions, contusions, or laceration. ${ }^{7,8}$ This is in relation with the biomolecular response to trauma: the release of excitatory amino acids is associated with calcium influx into neurons that consequently determines oxygen radical reaction ${ }^{27}$; additional sources of free radicals include the arachidonic acid cascade, mitochondria leakage, xanthine oxidase activity, and the autoxidation of amine neurotransmitters. ${ }^{28}$ When free radicals accumulate and the $\mathrm{pH}$ is lowered, the conditions are favorable for the deposition of iron, which subsequently keeps the inflammatory response triggered. It also has been shown that 
trauma-induced increased blood-brain barrier permeability ${ }^{9}$ and regional hemoglobin degradation products can be found in the absence of local lesions or distant to the site of primary insult, ${ }^{10}$ suggesting another mechanism for excessive iron deposition in TBI. In addition, a pathologic case report in a patient with mTBI who died unexpectedly showed a considerable amount of hemosiderin-laden macrophages in the frontal lobe, though both the CT and the gross macroscopic postmortem evaluation were negative. ${ }^{29}$ This is in line with findings in experimental models of brain trauma where diffuse brain hemosiderin deposits were present even when no gross detection of hemorrhage was identified, ${ }^{30}$ and with the demonstrated improvement in spatial memory performance in animal models of trauma after treatment with deferoxamine, an iron scavenger. ${ }^{31}$ Although any attempt to interpret the underlying substrate of MFC changes is speculative, and could only be confirmed by a postmortem examination, our findings of microstructural damage in basal ganglia and thalami in patients with mTBI are supported by studies using independent imaging techniques. We cannot rule out that the iron deposition in the thalamus and globus pallidus, suggested by our MFC measurements, is secondary to DAI, microhemorrhages, and microgliosis of the white matter tracts surrounding the deep gray matter structure. Similarly to what has been described in other neurologic diseases, ${ }^{32}$ it might be possible that due to the injury of axons that project to the cortex, the iron is still taken up by deep gray matter structures, but its centrifugal transportation to the cortex is impaired leading to local accumulation.

We did not find any correlations between the increase of MFC in the thalamus and in the globus pallidus and the PCSS, Stroop, and VFT test scores. Although Stroop and VFT can measure attention and processing speed and are widely used in the assessment of patients with TBI, they are mainly a measure of executive functions and hence were correlated with the MFC value of the frontal white matter. Future studies using a more comprehensive neuropsychological battery will help in understanding the clinical relevance of iron deposition in thalamus and basal ganglia.

We did not find increased MFC values in the examined white matter regions of patients with $\mathrm{mTBI}$; this can be partly explained by the fact that we evaluated only the frontal white matter and the splenium of the corpus callosum. Whereas the deep gray matter is sensitive to shear and strain damage due to its central location in the brain, the white matter injury is more dependent on the site of head injury, which varied in our patients. However, because we focused on the splenium of the corpus callosum, which is a site of predilection for DAI, the absence of detectable MFC changes also might be related to limitation of the technique used. For example, our sequence only measured the MFC at a single time point $(\mathrm{TE} / 2=20$ $\mathrm{ms}$ ) and would be insensitive to MFIs with a length scale small compared with the diffusion length $\left(3 \mathrm{D}^{\star} \mathrm{TE}\right)$ $1 / 2 \approx 7.7 / \mu \mathrm{m}$ (assuming a water diffusion coefficient of $\mathrm{D}=$ $\left.1 / \mu \mathrm{m}^{2} / \mathrm{ms}\right)$.

Finally, the cross-sectional design of our study limits our understanding of the timing and role of iron accumulation. Ongoing longitudinal studies in patients with acute mTBI will provide a better understanding of the pathophysiologic cascade of events leading to brain iron accumulation. The application of MFC MR imaging in neurologic diseases associated with well-known changes in tissue iron deposition ${ }^{12,13,15}$ supports its potential to provide insights into the pathophysiologic mechanisms of tissue injury in mTBI.

\section{Conclusions}

To the best of our knowledge, this is the first study investigating the presence of tissue iron deposition in patients with mTBI. Using a recently developed MR imaging technique, sensitive to the presence of iron deposits, we found significantly increased MFC values in the thalamus and globus pallidus of patients with mTBI, probably reflecting iron accumulation. Our findings corroborate the evidence of deep gray matter as a frequent site of injury in mTBI, and support the hypothesis of a possible role for iron accumulation in the pathophysiologic events after mTBI.

\section{Acknowledgments}

We thank Drs Ali Tabesh and Cathy Hu for helpful technical assistance.

Disclosures: Robert I. Grossman, Research Support (including provision of equipment or materials): National Institutes of Health grant R01 NS039135.

\section{References}

1. Brown AW, Leibson CL, Malec JF, et al. Long-term survival after traumatic brain injury: a population-based analysis. Neurorehabilitation 2004;19:37-43

2. Bazarian JJ, McClung J, Shah MN, et al. Mild traumatic brain injury in the United States, 1998-2000. Brain Injury 2005;19:85-91

3. Ropper AH, Gorson KC. Clinical practice. Concussion. N Engl J Med 2007;356: $166-72$

4. Thurman DJ, Alverson C, Dunn KA, et al. Traumatic brain injury in the United States: a public health perspective. J Head Trauma Rehabil 1999;14:602-15

5. Hammoud DA, Wasserman BA. Diffuse axonal injuries: pathophysiology and imaging. Neuroimaging Clin N Am 2002;12:205-16

6. Medana IM, Esiri MM. Axonal damage: a key predictor of outcome in human CNS diseases. Brain 2003;126:515-30

7. Bakay L, Lee JC, Lee GC, et al. Experimental cerebral concussion. Part 1: an electron microscopic study. J Neurosurg 1977;47:525-31

8. Jane JA, Steward O, Gennarelli TA. Axonal degeneration induced by experimental noninvasive minor head injury. J Neurosurg 1985;62:96-100

9. Adelson PD, Whalen MJ, Kochanek PM, et al. Blood brain barrier permeability and acute inflammation in two models of traumatic brain injury in the immature rat: a preliminary report. Acta Neurochir Suppl 1998;71:104-06

10. Povlishock JT. Traumatically induced axonal injury: pathogenesis and pathobiological implications. Brain Pathol 1992;2:1-12

11. Onyszchuk G, Levine SM, Brooks WM, et al. Post-acute pathological changes in the thalamus and internal capsule in aged mice following controlled cortical impact injury: a magnetic resonance imaging, iron histochemical, and glial immunohistochemical study. Neurosci Lett 2009;452:204-08

12. Jensen JH, Szulc K, Hu C, et al. Magnetic field correlation as a measure of iron-generated magnetic field inhomogeneities in the brain. Magn Reson Med 2009;61:481-85

13. Jensen JH, Chandra R, Ramani A, et al. Magnetic field correlation imaging. Magn Reson Med 2006;55:1350-61

14. Sukstanskii AL, Yablonskiy DA. Gaussian approximation in the theory of MR signal formation in the presence of structure-specific magnetic field inhomogeneities. J Magn Reson 2003;163:236-47

15. Ge Y, Jensen JH, Lu H, et al. Quantitative assessment of iron accumulation in the deep gray matter of multiple sclerosis by magnetic field correlation imaging. AJNR J Neuroradiol 2007;28:1639-44

16. Kay T, Harrington D, Adams R, et al. Definition of mild traumatic brain injury. J Head Trauma Rehabil 1993;8:86-87

17. Trenerry M, Crosson B, LeBoe J, et al. Stroop Neuropsychological Screening Test Manual. Odessa, Florida: Psychological Assessment Resources; 1989

18. Delis DC, Kramer JH, Kaplan E, et al. Reliability and validity of the DelisKaplan Executive Function System: an update. J Int Neuropsychol Soc 2004;10: 301-03

19. Aubry M, Cantu R, Dvorak J, et al. Summary and agreement statement of the First International Conference on Concussion in Sport, Vienna 2001. Recommendations for the improvement of safety and health of athletes who may suffer concussive injuries. Br J Sports Med 2002;36:6-10

20. Lull N, Noé E, Lull JJ, et al. Thalamic metabolism and neurological outcome 
after traumatic brain injury. A voxel-based morphometric FDG-PET study. Neurologia 2010;25:174-80

21. Lifshitz J, Kelley BJ, Povlishock JT. Perisomatic thalamic axotomy after diffuse traumatic brain injury is associated with atrophy rather than cell death. $\mathrm{JNeu}$ ropathol Exp Neurol 2007;66:218-29

22. Haber SN, Calzavara R. The cortico-basal ganglia integrative network: the role of the thalamus. Brain Res Bull 2009;78:69-74

23. Shaw NA. The neurophysiology of concussion. Prog Neurobiol 2002;67:281-344

24. Sipe JC, Lee P, Beutler E. Brain iron metabolism and neurodegenerative disorders. Dev Neurosci 2002;24:188-96

25. Connor JR, Menzies SL, Burdo JR, et al. Iron and iron management proteins in neurobiology. Pediatr Neurol 2001;25:118-29

26. Campbell A, Smith MA, Sayre LM, et al. Mechanisms by which metals promote events connected to neurodegenerative diseases. Brain Res Bull 2001;55: $125-32$
27. Greve MW, Zink BJ. Pathophysiology of traumatic brain injury. Mt Sinai JMed 2009;76:97-104

28. Hall ED, Vaishnav RA, Mustafa AG. Antioxidant therapies for traumatic brain injury. Neurotherapeutics 2010;7:51-61

29. Bigler ED. Neuropsychological results and neuropathological findings at autopsy in a case of mild traumatic brain injury. J Int Neuropsychol Soc 2004;10: $794-806$

30. Adelson PD, Jenkins LW, Hamilton RL, et al. Histopathologic response of the immature rat to diffuse traumatic brain injury. J Neurotrauma 2001;18: $967-76$

31. Long DA, Ghosh K, Moore AN, et al. Deferoxamine improves spatial memory performance following experimental brain injury in rats. Brain Res 1996;717: 109-17

32. Craelius W, Migdal MW, Luessenhop CP, et al. Iron deposits surrounding multiple sclerosis plaques. Arch Pathol Lab Med 1982;106:397-99 